\title{
The Existence and Stability of Synchronizing Solution of Non-Autonomous Equations with Multiple Delays
}

\author{
Jinying Wei' ${ }^{1}$, Yongjun $\mathrm{Li}^{1}$, Xiaohua Zhuo ${ }^{2}$ \\ ${ }^{1}$ School of Mathematics, Lanzhou City University, Lanzhou, China \\ ${ }^{2}$ Gansu Province Health School, Lanzhou, China \\ Email:weijy2818@163.com
}

Received 24 May 2016; accepted 12 July 2016; published 15 July 2016

\begin{abstract}
In this paper, we consider an abstract non-autonomous evolution equation with multiple delays in a Hilbert space $H: u^{\prime}(t)+A u(t)=F\left(u\left(t-r_{1}\right), \ldots, u\left(t-r_{n}\right)\right)+g(t)$, where $A: D(A) \subset H \rightarrow H$ is a positive definite selfadjoint operator, $F: H_{\alpha}^{n} \rightarrow H$ is a nonlinear mapping, $r_{1}, \ldots, r_{n}$ are nonnegative constants, and $g(t) \in C(\mathbb{R} ; H)$ is bounded. Motivated by [1] [2], we obtain the existence and stability of synchronizing solution under some convergence condition. By this result, we provide a general approach for guaranteeing the existence and stability of periodic, quasiperiodic or almost periodic solution of the equation.
\end{abstract}

\section{Keywords}

Pullback Attractor, Cocycle System, Stability, Synchronizing Solution

\section{Introduction}

In this paper, we consider the following non-autonomous evolution equation with multiple delays in a Hilbert space $H$ :

$$
u^{\prime}(t)+A u(t)=F\left(u\left(t-r_{1}\right), \ldots, u\left(t-r_{n}\right)\right)+g(t),
$$

where $A: D(A) \subset H \rightarrow H$ is a positive definite selfadjoint operator with compact resolvent, $F: H_{\alpha}^{n} \rightarrow H$ is a nonlinear mapping, $r_{1}, \ldots, r_{n}$ are nonnegative constants, and $g(t) \in C(\mathbb{R} ; H)$ is bounded.

This partial differential equations with delays (1.1) has extensive physical background and realistic mathematical model, hence it has been considerably developed and the numerous properties of their solutions have been studied, see [3]-[5] and references therein. Ref. [4] and [5] mainly discussed the existence and stability of periodic solutions of (1.1). Ref. [3] is concerned with the existence of locally almost periodic solutions of (1.1) by pullback attractor theory.

In this paper, our aim is to study the existence and stability of synchronizing solution of Equation (1.1). Mo- 
tivated by [1] [2], we obtain the existence and stability of synchronizing solution under some convergence condition. The result be of most interest when we choose $g(t) \in C_{b}(\mathbb{R}, H)$ be translation compact (resp. recurrent or almost periodic or quasiperiodic or periosdic), then we can obtain the synchronizing solution of Equation (1.1) is also translation compact (resp. recurrent or almost periodic or quasiperiodic or periosdic). This result provides a general approach for guaranteeing the existence and stability of periodic, quasiperiodic, almost periodic or recurrent solution of the equation.

The rest of the paper is organized as follows. In Section 2, we provide some preliminaries. In Section 3, we establish the existence and stability of synchronizing solutions under some convergence condition.

\section{Preliminaries}

This section consists of some preliminary work.

\subsection{Analytic Semigroups}

Let $H$ be a Hilbert space with the inner product $(\cdot, \cdot)$. We will use $|\cdot|$ to denote the norm of $H$ and use $\|\cdot\|$ to denote the norm of bounded linear operators on $H$. Let

$$
A: D(A) \subset H \rightarrow H
$$

be a positive definite selfadjoint operator with compact resolvent, and let

$$
0<\lambda_{1} \leq \lambda_{2} \leq \ldots \leq \lambda_{k} \rightarrow \infty \quad(k \rightarrow \infty)
$$

Be the eigenvalues of $A$ (counting with multiplicity) with the corresponding eigenvectors $\left\{\omega_{i}\right\}_{i=1}^{\infty}$ which form a canonical basis of $H$.

For $\alpha \in[0,1]$, define the powers $A^{\alpha}$ as follows:

$$
A^{\alpha} u=\sum_{i=1}^{\infty} \lambda_{i}^{\alpha} c_{i} \omega_{i}, \quad \forall u=\Sigma_{i=1}^{\infty} C_{i} \omega_{i} \in H .
$$

Let

$$
H_{\alpha}=D\left(A^{\alpha}\right):=\left\{u \in H \mid \Sigma_{i=1}^{\infty} \lambda_{i}^{\alpha} c_{i}^{2}<\infty\right\} .
$$

Then, $H_{\alpha}$ is a Hilbert space with the inner product $(\cdot, \cdot)_{\alpha}$ and norm $|\cdot|_{\alpha}$ defined as

$$
(u, v)_{\alpha}=\left(A^{\alpha} u, A^{\alpha} v\right),|u|_{\alpha}=(u, u)_{\alpha}^{1 / 2}, \quad \forall u, v \in H_{\alpha},
$$

respectively. We also know that for any $0 \leq \alpha<\beta \leq 1$, the embedding $H_{\beta} \subset H_{\alpha}$ is compact; moreover, it holds that

$$
|v|_{\alpha}^{2} \leq \lambda_{1}^{2(\alpha-\beta)}|v|_{\beta}^{2}, \quad \forall v \in H_{\beta} .
$$

\subsection{Pullback Attractors}

We recall some basic definitions and facts in the theory of non-autonomous dynamical systems for skew-product flows on complete metric spaces.

Let $(X, d)$ be a complete metric space, $(\Sigma, \rho)$ be a metric space which will be called the base space (or symbol space). $\theta: \mathbb{R} \times \Sigma \rightarrow \Sigma$ is a mapping, $\theta_{t}=\theta(t, \cdot): \Sigma \rightarrow \Sigma$ form a group, that is, $\theta$ satisfies

1) $\theta_{0}=i d_{\Sigma}$

2) $\theta_{t+s}=\theta_{t} \cdot \theta_{s}, \forall t, s \in \mathbb{R}$.

Definition 2.1 A mapping $\Phi: \mathbb{R}^{+} \times \Sigma \times X \rightarrow X$ is said to be a continuous cocycle on $X$ with respect to group $\theta$, if

1) $\Phi(0, \sigma, x)=x, \forall(\sigma, x) \in \Sigma \times X$;

2) $\Phi(t+s, \sigma, x)=\Phi\left(t, \theta_{s} \sigma, \Phi(s, \sigma, x)\right), \forall t, s \geq 0$ and $(\sigma, x) \in \Sigma \times X$;

3) $\Phi: R^{+} \times \Sigma \times X \rightarrow X$ is continuous.

The mapping $\pi: R^{+} \times \Sigma \times X \rightarrow \Sigma \times X$ defined by

$$
\pi(t, \sigma, x):=\left(\theta_{t} \sigma, \Phi(t, \sigma, x)\right), \forall t \in R,(\sigma, x) \in \Sigma \times X,
$$

forms a semigroup on $\Sigma \times X$ and is called a skew-product flow. 
Definition 2.2 A family $\mathcal{A}=\{A(\sigma)\}_{\sigma \in \Sigma}$ of nonempty compact sets of $X$ is called a global pullback attractor of the cocycle $\Phi$ if it is $\Phi$-invariant, that is,

$$
\Phi(t, \sigma, A(\sigma))=A\left(\theta_{t} \sigma\right), \forall t \in R^{+}, \sigma \in \Sigma,
$$

and pullback attracting, that is, for any bounded subset $B$ of $X$,

$$
\lim _{t \rightarrow+\infty} d_{H}\left(\Phi\left(t, \theta_{-t} \sigma, B\right), A_{\sigma}\right)=0, \forall \sigma \in \Sigma,
$$

and is the minimal family of compact sets that is both invariant and pullback attracting.

\subsection{Global Pullback Attractor of (1.1)}

We present essential conditions on the nonlinearity $F$ to guarantee the dissipation and the existence of pullback attractor of (1.1).

We first discuss the well-posedness of the initial value problem of the equation.

Let $C=C\left([-r, 0] ; H_{\alpha}\right)$, where $r=\max \left\{r_{1}, \ldots, r_{n}\right\}$. $C$ is endowed with the norm

$$
\|u\|_{C}=\max _{t \in[-r, 0]}|u(t)|_{\alpha} .
$$

For $u \in C$ and $t \in[0, \infty)$, we define $u_{t} \in C$ by

$$
u_{t}(s)=u(t+s), \quad s \in[-r, 0] .
$$

Consider the initial value problem of the evolution equation with delays

$$
\left\{\begin{array}{l}
\frac{d}{d t} u(t)+A u(t)=F\left(u\left(t-r_{1}\right), \ldots, u\left(t-r_{n}\right)\right)+g(t), t>0, \\
u_{0}=\varphi, \varphi \in C .
\end{array}\right.
$$

where $F: H_{\alpha}^{n} \rightarrow H$ is continuous and there exist positive constants $\beta_{1}, \ldots, \beta_{n}$ and $k_{1}$ such that $F$ satisfies the following conditions:

(H1) For all $\left(v_{1}, \ldots, v_{n}\right) \in H_{\alpha}^{n}$ and $t \in \mathbb{R}^{1}$

$$
\left|F\left(v_{1}, \ldots, v_{n}\right)\right| \leq \sum_{i=1}^{n} \beta_{i}\left|v_{i}\right|_{\alpha}+k_{1} ;
$$

(H2) $\sum_{i=1}^{n} \beta_{i}<\lambda_{1}^{1-\alpha}$;

(H3) For any $R>0$ and bounded interval $J$, there exists $L>0$ such that

$$
\left|F\left(v_{1}, \ldots, v_{n}\right)-F\left(w_{1}, \ldots, w_{n}\right)\right| \leq \sum_{i=1}^{n} L\left|v_{i}-w_{i}\right|_{\alpha}
$$

for all $v_{i}, w_{i} \in B_{\alpha}(0, R)$ and $t \in J$, where (and hereafter) $B_{\alpha}(0, R)$ denotes the ball in $H_{\alpha}$ centered at 0 with radius $R$;

and $g(t) \in C(\mathbb{R} ; H)$ is bounded, that is, there exists a positive constant $k_{2}>0$ such that for all $t \in \mathbb{R}$

$$
|g(t)| \leq k_{2} .
$$

Theorem 2.3 Assume that $0 \leq \alpha \leq 1 / 2$ and $F$ satisfies (H1)-(H3). Then, the problem (2.2) has a unique global mild solution $u(t)=u(t ; g, \varphi)$ which depends on $\varphi$ continuously, and

$$
\begin{gathered}
u(\cdot) \in C\left([-r, \infty) ; H_{\alpha}\right) \cap L_{l o c}^{2}\left(0, \infty ; H_{1}\right), \\
\frac{d}{d t} u(\cdot) \in L_{l o c}^{2}\left(0, \infty ; H_{\alpha-1 / 2}\right) .
\end{gathered}
$$

Proof. The proof can be obtained by Theorem 5 in [3].

Remark 2.4 u satisfies the following integral equation:

$$
u(t)=T(t) \varphi(0)+\int_{0}^{t} T(t-s) F\left(u\left(s-r_{1}\right), \ldots, u\left(s-r_{n}\right)\right)+T(t-s) g(s) d s .
$$

Let the space $C\left(\mathbb{R}^{1} ; H\right)$ be equipped with the compact-open topology: 


$$
\rho(u, v)=\sum_{n=1}^{\infty} \frac{1}{2^{n}} \frac{\max _{|t| \leq n}|u(t)-v(t)|_{\alpha}}{1+\max _{|t| \leq n}|u(t)-v(t)|_{\alpha}}, \quad \forall u, v \in C\left(\mathbb{R}^{1} ; H\right) .
$$

It is well known that this topology is metrizable and $C\left(\mathbb{R}^{1} ; H\right)$ is a complete metric space.

Give $g(t) \in C\left(\mathbb{R}^{1} ; H\right)$ is bounded, we define the base space $\Sigma$ as

$$
\Sigma=\text { the closure of }\left\{g(t+\cdot) \mid t \in \mathbb{R}^{1}\right\} \text { in the metric } \rho \text {. }
$$

So the shift operator $\theta=\theta_{t}: \Sigma \rightarrow \Sigma$ defined for each $t \in \mathbb{R}$ by

$$
\theta_{t} \sigma=\sigma(t+\cdot), \forall \sigma \in \Sigma,
$$

forms a continuous dynamical system on the base space $\Sigma$.

Define $\Phi: \mathbb{R}^{+} \times \Sigma \times C \rightarrow C$ as follows:

$$
\Phi(t, \sigma, \varphi)=u_{t}, \quad \forall(t, \sigma, \varphi) \in \mathbb{R}^{+} \times \Sigma \times C,
$$

where $u(t)=u(t ; \sigma, \varphi)$ is the unique solution of the problem (2.2) with $g=\sigma$. Then $\Phi$ is a cocycle system on $C$ with the base space $\Sigma$ and driving system $\theta$.

Since Theorem 12 in [3], we have the following existence result concerning the pullback attractors.

Theorem 2.5 Let $0 \leq \alpha \leq 1 / 2$. If $F$ satisfies conditions (H1)-(H3), then $\Phi$ has a unique global pullback attractor $\mathcal{A}=\{A(\sigma)\}_{\sigma \in \Sigma}$.

\section{Synchronizing Solutions}

In this section, we establish some results on synchronizing solutions for (1.1), by developing some techniques inspired by works [2] and [1]. It is known that if g has some special structure, i.e., periodic, quasiperiodic, almost periodic etc., then we can obtain a compact base space with same structure. Combined with the theory of uniform pullback attractors for dynamical systems in [6], we will prove that under some convergence condition, Equation (1.1) have some entire solution $\gamma(t)$ that synchronize with the motion of the driving system. We call $\gamma(t)$ synchronizing solutions for (1.1).

Now, we consider that $g(t) \in C\left(\mathbb{R}^{1}, H\right)$ is translation compact, then the base space $\Sigma=H(g)$ is compact.

If furthermore, the Lipschitz coefficients $L_{i}$ of $F$ in the set $B_{H_{\alpha}}\left(0, \rho_{\alpha}\right)$ satisfy:

$$
\sum_{i=1}^{n} L_{i}<\lambda_{1}^{1-\alpha} \text {, (H4) }
$$

then we have the following results about synchronizing solutions for (1.1).

Theorem 3.1 Assume $0 \leq \alpha \leq 1 / 2$, and $F$ satisfies (H1)-(H4). Let $g(t)$ is translation compact in $C\left(\mathbb{R}^{1} ; H\right)$. Then:

1) There exists a $\Gamma \in C(\Sigma)$ such that for each $\sigma \in \Sigma, \gamma_{\sigma}(t):=\Gamma\left(\theta_{t} \sigma\right)$ is the unique bounded entire solution of (1.1) on $\mathbb{R}$;

2) For any $\varphi \in C$, there exists a unique solution $u(t ; \sigma, \varphi)$ of $(1.1)$ on $\mathbb{R}^{+}$with initial value $u_{0}=\varphi$ that satisfies

$$
\lim _{t \rightarrow+\infty} \sup _{\sigma \in \Sigma}\left|u(t ; \sigma, \varphi)-\gamma_{\sigma}(t)\right|_{\alpha}=0 .
$$

Proof. By Theorem 2.5, we have proved that the cocycle mapping $\Phi$ has a pullback attractor $\mathcal{A}=\{A(\sigma)\}_{\sigma \in \Sigma}$, and we know that $\mathcal{A} \subset U$ is bounded. So $\mathcal{A}$ is given as the union all bounded entire solution.

As Definition 2.2, it is $\Phi$-invariant, that is,

$$
\Phi(t, \sigma, A(\sigma))=A\left(\theta_{t} \sigma\right), \quad \forall t \in \mathbb{R}^{+}, \sigma \in \Sigma,
$$

One can also write the non-autonomous invariance property as

$$
\Phi\left(t, \theta_{-t} \sigma, A\left(\theta_{-t} \sigma\right)\right)=A(\sigma), \quad \forall t \in \mathbb{R}^{+}, \sigma \in \Sigma .
$$

In what follows we show that for each $\sigma \in \Sigma, A(\sigma)$ is in fact a singleton, i.e.,

$$
A(\sigma)=\left\{a_{\sigma}\right\}
$$

for some $a_{\sigma} \in C$. 
Let $\varphi, \psi \in A(\sigma)$. By invariance property (3.1), for any $\tau>0$ there exist $\varphi_{1}, \psi_{1} \in A\left(\theta_{-\tau} \sigma\right)$ such that

$$
\Phi\left(\tau, \theta_{-\tau} \sigma, \varphi_{1}\right)=\varphi, \quad \Phi\left(\tau, \theta_{-\tau} \sigma, \psi_{1}\right)=\psi .
$$

We know that

$$
\Phi\left(\tau, \theta_{-\tau} \sigma, \varphi_{1}\right)=u_{\tau}=u\left(\tau+s ; \theta_{-\tau} \sigma, \varphi_{1}\right),
$$

where $u\left(; \theta_{-\tau} \sigma, \varphi_{1}\right)$ is the solution of (2.2) with initial value $\varphi_{1}$, and

$$
\Phi\left(\tau, \theta_{-\tau} \sigma, \psi_{1}\right)=v_{\tau}=u\left(\tau+s ; \theta_{-\tau} \sigma, \psi_{1}\right),
$$

where $u\left(; \theta_{-\tau} \sigma, \psi_{1}\right)$ is the solution of (2.2) with initial value $\psi_{1}$.

Let $w(t)=u(t)-v(t)=u\left(t ; \theta_{-\tau} \sigma, \varphi_{1}\right)-u\left(t ; \theta_{-\tau} \sigma, \psi_{1}\right)$, we have

$$
w^{\prime}(t)+A w(t)=F\left(u\left(t-\tau_{1}\right), \ldots, u\left(t-\tau_{n}\right)\right)-F\left(v\left(t-\tau_{1}\right), \ldots, v\left(t-\tau_{n}\right)\right) .
$$

Taking inner product with $A^{2 \alpha} w(t)$ and using Hölder's inequality, (H3), Poincáre’s inequality and Young's inequality, we have

$$
\frac{1}{2} \frac{d}{d t}|w(t)|_{\alpha}^{2}+|w(t)|_{\frac{1}{2}+\alpha}^{2} \leq\left(\sum_{i=1}^{n} L_{i}\left|w\left(t-r_{i}\right)\right|_{\partial}\right) \cdot|w(t)|_{2 \alpha} \leq \frac{\lambda_{1}^{\alpha}}{2} \sum_{i=1}^{n} L_{i}\left|w\left(t-r_{i}\right)\right|_{\varepsilon}^{2}+\frac{\lambda_{1}^{-\alpha}}{2} \sum_{i=1}^{n} L_{i}|w(t)|_{2 \alpha}^{2},
$$

which yields that

$$
\frac{d}{d t}|w(t)|_{\alpha}^{2} \leq-\eta_{1}|w(t)|_{\alpha}^{2}+\sum_{i=1}^{n} \lambda_{1}^{\alpha} L_{i}\left|w\left(t-r_{i}\right)\right|_{\alpha}^{2},
$$

where $\eta_{1}=\lambda_{1}^{\alpha}\left(2 \lambda_{1}^{1-\alpha}-\sum_{i=1}^{n} L_{i}\right)$. Integrating from 0 to $t$, we obtain

$$
|w(t)|_{\alpha}^{2}-|w(0)|_{\alpha}^{2} \leq-\eta_{1} \int_{0}^{t}|w(s)|_{\alpha}^{2} d s+\sum_{i=1}^{n} \lambda_{1}^{\alpha} L_{i} \int_{0}^{t}\left|w\left(s-r_{i}\right)\right|_{\alpha}^{2} d s \leq-2 \hat{\eta} \int_{0}^{t}|w(s)|_{\alpha}^{2} d s+\sum_{i=1}^{n} \lambda_{1}^{\alpha} L_{i} \int_{-r_{i}}^{0}|w(s)|_{\alpha}^{2} d s,
$$

where $\hat{\eta}=\lambda_{1}^{\alpha}\left(\lambda_{1}^{1-\alpha}-\sum_{i=0}^{n} L_{i}\right)$.

Let $\gamma_{1}^{2}=1+\sum_{i=1}^{n} \lambda_{1}^{\alpha} L_{i} r_{i}$. We obain

$$
|w(t)|_{\alpha}^{2}+2 \hat{\eta} \int_{0}^{t}|w(s)|_{\alpha}^{2} d s \leq \gamma_{1}^{2}\left\|\varphi_{1}-\psi_{1}\right\|_{c}^{2} .
$$

Since $\mathcal{A} \subset U$ and (H4), we have $\hat{\eta}>0$. Then by Gronwall's lemma we have

$$
|u(t)-v(t)|_{\alpha} \leq \gamma_{1} e^{-\hat{\eta} t}\left\|\varphi_{1}-\psi_{1}\right\|_{C}=2 \gamma_{1} \rho_{\alpha} e^{-\hat{\eta} t} .
$$

Then, we can obtain that

$$
\|\varphi-\psi\|_{C} \max _{s \in[-r, 0]}\left|u\left(\tau+s ; \theta_{-\tau} \sigma, \varphi_{1}\right)-u\left(\tau+s ; \theta_{-\tau} \sigma, \psi_{1}\right)\right|_{\alpha} \leq 2 \gamma_{1} \rho_{\alpha} e^{-\hat{\eta}(\tau-r)},
$$

which implies $\|\varphi-\psi\|_{C} \rightarrow 0$ as $\tau \rightarrow+\infty$. Hence, $\varphi=\psi$.

Now define $\Gamma: \Sigma \rightarrow H$ as

$$
\Gamma(\sigma)=A_{\sigma}(0), \quad \forall \sigma \in \Sigma .
$$

We infer from Corollary 2.8 in [6] that $A(\sigma)$ is upper semi-continuous in $\sigma$. This reduces to the continuity of $a_{\sigma}$ in $\sigma$ when the $\{A(\sigma)\}$ are single point sets. Hence, $\Gamma$ is continuous. For each $\sigma \in \Sigma$, set

$$
\gamma_{\sigma}(t)=\Gamma\left(\theta_{t} \sigma\right), \quad t \in \mathbb{R} .
$$

By invariance property of $\mathcal{A}$ one trivially checks that $\gamma_{\sigma}$ is precisely the unique solution of (1.1) on $\mathbb{R}$. Since Theorem 4.3 in [5], $\mathcal{A}$ is a uniform pullback attractor. That is, for any $\varphi \in \mathcal{C}$, we have

$$
\lim _{t \rightarrow+\infty} \sup _{\sigma \in \Sigma} H_{C}^{*}\left(\Phi\left(t, \theta_{-t} \sigma, \varphi\right), A(\sigma)\right)=0,
$$

where $H_{C}^{*}(\cdot, \cdot)$ denotes the semi-Hausdorff distance in $C$. Then, it is uniformly forwards attracting, 


$$
\lim _{t \rightarrow+\infty} \sup _{\sigma \in \Sigma} H_{C}^{*}\left(\Phi(t, \sigma, \varphi), A\left(\theta_{t} \sigma\right)\right)=0 .
$$

Thus we can deduce that

$$
\lim _{t \rightarrow+\infty} \sup _{\sigma \in \Sigma}\left|u(t ; \sigma, \varphi)-\gamma_{\sigma}(t)\right|_{\alpha}=0 .
$$

The proof is complete.

Corollary 3.2 Let $g \in C\left(\mathbb{R}^{1}, H\right)$ is periodic (resp. quasiperiodic, almost periodic, recurrent), then under conditions of Theorem 3.1 the non-autonomous Equation (1.1) admits a unique periodic (resp. quasiperiodic, almost periodic, recurrent) solution $\gamma_{\sigma}(t)$ and every other solution of this equation are asymptotically periodic (resp. asymptotically quasiperiodic, asymptotically almost periodic, asymptotically recurrent).

Proof. Let $\gamma_{\sigma}(t)=\Gamma\left(\theta_{t} \sigma\right)$ be a function from Theorem 3.1, then according to this theorem we conclude that $\gamma_{\sigma}(t)$ is the unique bounded solution of (1.1) synchronizing with the motion $\theta_{t} \sigma$ of the driving system $\theta$. In particular, if $\theta_{t} \sigma$ is periodic (resp. quasiperiodic, almost periodic, recurrent), then so is $\gamma_{\sigma}$. By (2) of Theorem 3.1, we know that every other solution of this equation is asymptotically periodic (resp. asymptotically quasiperiodic, asymptotically almost periodic, asymptotically recurrent). The proof is complete.

\section{Acknowledgements}

This work was supported by NNSF (11261027), NNSF (11161026) and the Research Funds of Lanzhou City University (LZCU-BS2015-01).

\section{References}

[1] Bongolan-Walsh, V.P., Cheban, D. and Duan, J. (2003) Recurrent Motions in the Nonautonomous Navier-Stokes System. Discrete and Continuous Dynamical Systems-Series B, 3, 255-262. http://dx.doi.org/10.3934/dcdsb.2003.3.255

[2] Li, D. and Duan, J. (2009) Structure of the Set of Bounded Solutions for a Class of Nonautonomous Second-Order Differential Equations. Journal of Differential Equations, 246, 1754-1773. http://dx.doi.org/10.1016/j.jde.2008.10.031

[3] Li, D., Wei, J. and Wang, J. (2013) On the Dynamics of Abstract Retarded Evolution Equations. Abstract and Applied Analysis, 2013, 9 p.

[4] Li, Y. (2011) Existence and Asymptotic Stability of Periodic Solution for Evolution Equations with Delays. Journal of Functional Analysis, 261, 1309-1324. http://dx.doi.org/10.1016/j.jfa.2011.05.001

[5] Zhu, J., Liu, Y. and Li, Z. (2008) The Existence and Attractivity of Time Periodic Solutions for Evolution Equations with Delays. Nonlinear Analysis: Real World Applications, 9, 842-851. http://dx.doi.org/10.1016/j.nonrwa.2007.01.004

[6] Cheban, D., Kloeden, P.E. and Schmalfuss, B. (2002) The Relationship between Pullback, Forwards and Global Attractors of Nonautonomous Dynamical Systems. Nonlinear Dynamics and Systems Theory, 2, 9-28.

\section{Submit or recommend next manuscript to SCIRP and we will provide best service for you:}

Accepting pre-submission inquiries through Email, Facebook, LinkedIn, Twitter, etc. A wide selection of journals (inclusive of 9 subjects, more than 200 journals)

Providing 24-hour high-quality service

User-friendly online submission system

Fair and swift peer-review system

Efficient typesetting and proofreading procedure

Display of the result of downloads and visits, as well as the number of cited articles

Maximum dissemination of your research work

Submit your manuscript at: http://papersubmission.scirp.org/ 